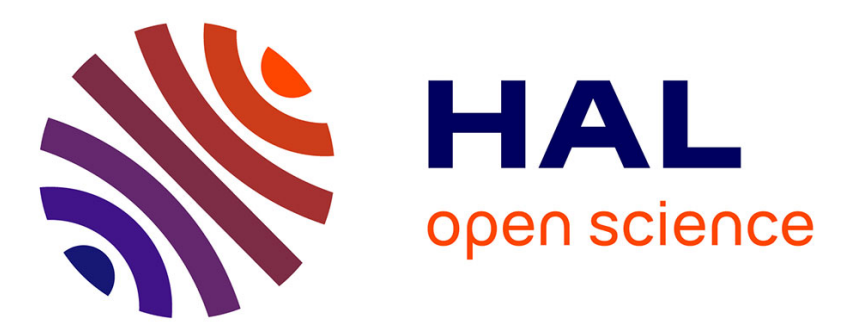

\title{
Quantum molecular dynamic approach to modeling the vibrational spectra of propynenitrile and its 1:1 complexes with water \\ Claude Pouchan
}

\section{- To cite this version:}

Claude Pouchan. Quantum molecular dynamic approach to modeling the vibrational spectra of propynenitrile and its 1:1 complexes with water. Computational and Theoretical Chemistry, 2019, 1162, pp.112499. 10.1016/j.comptc.2019.06.004 . hal-02288751

\section{HAL Id: hal-02288751 \\ https://hal.science/hal-02288751}

Submitted on 25 Oct 2021

HAL is a multi-disciplinary open access archive for the deposit and dissemination of scientific research documents, whether they are published or not. The documents may come from teaching and research institutions in France or abroad, or from public or private research centers.
L'archive ouverte pluridisciplinaire HAL, est destinée au dépôt et à la diffusion de documents scientifiques de niveau recherche, publiés ou non, émanant des établissements d'enseignement et de recherche français ou étrangers, des laboratoires publics ou privés.

\section{(c)(1) $\$$}

Distributed under a Creative Commons Attribution - NonCommerciall 4.0 International 


\section{Quantum molecular dynamic approach to modeling the vibrational spectra of propynenitrile and its 1:1 complexes with water.}

\section{Claude POUCHAN}

CNRS, UMR 5254, Institut des Sciences Analytiques et de Physico-Chimie pour l'Environnement et les Matériaux, Université de Pau et des Pays de l’Adour, F-64000 Pau, France.

*corresponding author: 00335594078 50,

claude.pouchan@univ-pau.fr

ORCID Number: 0000-0003-3841-4922

\section{Abstract}

We present here a quantum molecular dynamic approach from DFT molecular dynamics (DFT/MD) trajectories around $150 \mathrm{~K}, 300 \mathrm{~K}, 450 \mathrm{~K}$ and $600 \mathrm{~K}$ to compute the IR spectra of the propynenitrile (or cyanoacetylene) molecule $\mathrm{HC}_{3} \mathrm{~N}$, the simplest linear cyanopolyyne detected in interstellar clouds and circumstellar envelopes of stars. The DFT/MD results in fair agreement with the experimental data for the isolated molecule are close to those obtained, for all the valence modes, from an effective second order treatment (VPT2) at the CCSD(T) level of theory. This DFT/MD simulation is used to predict the IR spectra of the 1:1 complexes with water and analyse the shifts expected for the micro-hydrated molecule.

Keywords: Density functional theory; DFT Molecular Dynamic Simulation; IR spectroscopy; propynenitrile or cyanoacetylene molecules; complexes 1:1 with water 


\section{Introduction}

Vibrational spectroscopies (Infrared and Raman) and IR spectroscopy particularly are commonly used in laboratories to identify and know the quality of a product. To do this, it is sufficient to choose the most intense characteristic bands without worrying about the globality and the complexity of all the signals appearing in the spectrum. Indeed, all the IR bands of a molecule are not easy to assign because we can find in addition to fundamental bands, overtones and combinations whose position and intensity varies according to the physical state of the product which can be gaseous, liquid, in solution, solid or trapped in a matrix [1]. To help in the full identification of an IR spectrum, and to take into account the effects of the environment, modelling through theoretical chemistry methods must be used very often [2]. When we are only interested in the fundamental bands, quantum mechanics methods easily provide harmonic frequencies that are corrected by scale factors to take into account the anharmonicity and thus get closer the calculated spectrum to the observed data [3]. When we want to know in order to explain or predict the whole spectrum (fundamentals, overtones, combinations) and to account for the effects due to the environment (liquid, solution, solid, adsorption...) we must use much more sophisticated quantum methods. Two main types of methods make it possible to obtain a theoretical IR spectrum that can be classified into 'static' and 'dynamic' methods and whose main characteristics are briefly recalled in the paragraph 'Methods and Computational Details'

Several 'static' models have been developed and implemented to explicitly take into account anharmonicity. In particular, for small molecules converged rovibrational levels can be obtained by fully variational treatments, as Vibrational Configuration Interaction (VCI) [4-9] or (VCI-P) [10-12] methods when the configuration interaction is guessed by a perturbative treatment and more recently Vibrational Coupled Cluster method (VCC) $[13,14]$. However, to conveniently handle large isolated systems it becomes unavoidable to introduce some approximations concerning both the form of the potential and the rovibrational treatment .Today to modelling vibrational spectra in the mid infrared region the most successful approaches are based on truncated three-mode potentials by using the effective second order perturbative (VPT2) [15] treatment, fast and easy, which tends to correct the numerical instabilities of a traditional perturbative approach due to the presence of random degeneracy.

Beside these static approach and starting from the pioneer work of Wilson et al.[16], vibrational spectra can be calculated in an alternative way using a 'molecular dynamic' approach and statistical mechanic models based on the Fourier transform analysis of the time correlation function of atomic dipole moments. These molecular dynamic (MD) approaches in principle yield to a full description of the modes activated at a given energy or 
temperature and describe their couplings and finally allow the complete description of the IR properties as position and intensity for all bands. Although several quantum corrections to the classical time-correlation functions have been proposed, they don't affect the calculated wavenumbers but only the shape and the width of the bands [17].

This work aims to compare the spectra obtained by the two approaches for an isolated molecule and a molecule interacting with water in order to describe, as a first approximation, the effects of the environment related to micro-hydration. For MD simulations, DFT has been applied for the description of the electronic structure and gradients 's computations using the very popular Becke three parameter hybrid functional (B3LYP) [18] and a localized Gaussian type basis set [6-31+G(p,d)] known to give excellent IR spectra for organic molecules [19]. The DFT/MD results based on the propagation of the density matrix will be compared, for the valence modes, to the computed data obtained at CCSD(T)/aug-cc-pVTZ anharmonic wavenumbers issued from a VPT2 treatment also known to provide excellent results in the static method when the couplings between the normal modes are not very important.

The molecule chosen for this study is a linear system the propynenitrile $\mathrm{HC}_{3} \mathrm{~N}$ since it is the simplest cyanopolyyne discovered in interstellar environments. Detected in the atmosphere of Titan it was identified by means of the knowledge of the fundamental bands and has been extensively studied by means of careful laboratory studies of high resolution infra-red spectra since 1990 [20-24]. Recently, Bizzochi et al. [25] reinvestigate the IR spectrum up to $1100 \mathrm{~cm}^{-1}$ and improve all the data by an extensive list of highly accurate rest frequencies. Taking into account these experimental data, our objective will be to know what is the most appropriate approach to modelling the experimental spectrum.

After a brief description of the computational methods applied (section 2), the DFT/MD results obtained for the propynenitrile will be discussed and compared both with the experimental data and the results issued from $\mathrm{CCSD}(\mathrm{T}) / \mathrm{VPT} 2$ computations. A first approach of the IR spectra for the complexes $\mathrm{HC}_{3} \mathrm{~N}: \mathrm{H}_{2} \mathrm{O}$ will complete this theoretical study. (section 3).

\section{Methods and Computational details}

Structural optimization, harmonic frequencies, IR intensities, and anharmonic frequencies issuing from second order perturbative approach (VPT2) and ab-initio molecular dynamic simulations were performed with Gaussian 
09 program [26]. Spectral analysis from the MD simulations was obtained using the VAMD (Vibrational Asignments in Molecular Dynamic) program developed in our group and described in reference [27].

Electronic structure calculations were performed at the DFT level using the most common three parameter hybrid functional of Becke (B3LYP) [18] and a valence double zeta Pople basis set including diffuse and polarization functions, $6-31+\mathrm{G}(\mathrm{d}, \mathrm{p})$. This model chemistry was chosen since it has been previously shown that, for the prediction of harmonic and anharmonic force constants and in the case of small organic [18,28,29] and radical [30], systems it is able to recover the results obtained using the more expensive $\operatorname{CCSD}(\mathrm{T})$ [31]/Aug-ccpVTZ [32] approach.

\subsection{Static treatment of anharmonicity}

The static approach used to compute anharmonic effects using the perturbative (VPT2) approach is briefly recalled. From a minimum energy structure of a given system, a quartic force field is built in which the third and fourth derivatives are computed by $6 \mathrm{~N}-11$ ( $\mathrm{N}=$ number of atoms) numerical differentiations of analytical or numerical (in case of CCSD (T) calculations) second derivatives from a least square fitting procedure [33]. In the quartic approximation, the potential is given by:

$$
V=\frac{1}{2 !} \sum_{i} \omega_{i} q_{i}^{2}+\sum_{i, j, k} k_{i j k} q_{i} q_{j} q_{k}+\sum_{i, j, k, l} k_{i j k l} q_{i} q_{j} q_{k} q_{l}
$$

where $q_{i}$ the $i$-th dimensionless normal coordinate, and $\omega_{i}, k_{i j k}, k_{i j k l}$ the harmonic, cubic and quartic force constant respectively expressed in $\mathrm{cm}^{-1}$. Note that only the anharmonic force constants involving the tri-mode interactions $(\mathrm{k}=\mathrm{l})$ are determined by numerical differentiation.

Next, the vibrational frequencies can be evaluated in the VPT2 approach by using the well-know formula of Willets and coworkers [34] derived from second order perturbation theory:

From a technical point of view, it can be shown [35] that the best setup is obtained using a step size of $0.01 \AA$ for the numerical differentiation of harmonic frequencies. For DFT computations very tight convergence criteria for structural optimizations and fine grids for integral evaluation (that is at least 99 radial and 590 angular points) are necessary.

\subsection{Vibrational analysis from ab-initio Molecular dynamic simulations.}

Ab-initio molecular dynamic simulations were performed using the Atom-centered Density Matrix (ADMP) formalism [36-38] which uses localized Gaussian basis sets, thus allowing for sampling on the same potential used for the static methods. This is of particular importance when both the potential energy surface and the 
observables are sensitive to the choice of the DFT computational level and, in particular, it allows for the use of hybrid functionals [18, 39-41]. Furthermore, in our case, this choice let to a direct comparison of the results of anharmonic calculations obtained from static and dynamic approaches. Molecular dynamic simulations were performed in the microcanonical subspace [42] with a time-step of 0.2 fs over an overall period of $10 \mathrm{ps}$, the initial nuclear velocities being set to perform simulations averaging the temperatures of $150 \mathrm{~K}, 300 \mathrm{~K}, 450 \mathrm{~K}$ and 600K. A suite of programs developed in our lab is used to perform the spectral analysis from ADMP DFT trajectories [27]. Several papers report in detail a simple procedure [43, 44] to get the IR spectrum as Fourier transform of the dipole autocorrelation function [45] that is:

$I(\bar{v}) \propto \int d t\left\langle\dot{M}_{0} \dot{M}_{t}\right\rangle e^{i \omega t}$

M being the total dipole moment, $\dot{M}$ the derivative $\frac{d M}{d t}$ and $I(\bar{v})$ the intensity at the $\bar{v}$ wavenumber.

To assign the vibrational bands we used the Fourier Transform of Time Correlation Function (FTTCF) of the velocity $(\mathrm{V}(\mathrm{t}))$ obtained together with the dipole moment at each time step of the simulation, this function projected along the normal modes or better the internal or local modes giving as described in reference [27] a good representation of the band assignment.

This procedure has been used in our computational strategy to modelling successfully DFT/MD infra-red spectra for gaseous and micro-hydrated forms of nucleobases [46-50]

\section{Results and discussions}

\section{3-1 Propynenitrile as exemple of linear molecule.}

At B3LYP/6-31 G+(d,p) and CCSD(T)/Aug-cc-pVTZ levels the calculated geometries are very similar for $\mathrm{C}_{1}-\mathrm{C}_{2}$ (1.211 $\AA$ vs $1.204 \AA)$ and $\mathrm{C}_{2}-\mathrm{H}_{3}(1.067 \AA$ vs $1.063 \AA)$. A small difference of about 0.01 angstroms can be noted for the other two lengths $\mathrm{C}_{1}-\mathrm{C}_{4}(1.373 \AA$ vs $1.386 \AA)$ and $\mathrm{C}_{4}-\mathrm{N}_{5}(1.167 \AA$ vs 1.157$) \AA$. It should be emphasized the weak influence of the triple excitation at CCSD level for the computed bond lengths.

Table 1 reports the harmonic vibrational wavenumbers obtained at different levels of theory. The harmonic values obtained at the CCSD and DFT levels for the valence vibrations $\mathrm{v}_{1}, \mathrm{v}_{2}, \mathrm{v}_{3}, \mathrm{v}_{4}$ and the CCH bending mode $\mathrm{v}_{5}$ are in fairly good agreement. However, it should be noted that for $\mathrm{v}_{7}$ and $\mathrm{v}_{6}$ modes, the B3LYP values are overestimated by about 12 and $16 \%$ respectively compared to CCSD frequencies. Except for the modes $\mathrm{v}_{5}$ and 
$\mathrm{v}_{1}$ (this latter associated with the $\mathrm{CH}$ valence mode), the introduction of triple excitations reduces harmonic frequencies by 3 to $5 \%$, bringing them closer to the experimental data.

Table 2 reports the anharmonic vibrational values obtained for the five modes $v_{1}, v_{2}, v_{3}, v_{4}$ and $v_{5}$ from VPT2 and DFT/MD approaches. At VPT2 level, modes $\mathrm{v}_{6}$ and $\mathrm{v}_{7}$ that have large differences according to their CCSD or B3LYP harmonic determination are decoupled from the other five modes to compute anharmonic wavenumbers. In addition, it should be noted that the quartic force constants are not often adapted for the description of the soft modes that are here $v_{6}$ and $v_{7}$ leading to inappropriate VPT2 results to describe these modes. As reported by Handy and coworkers [51], the simplest VPT2 treatment generally provides, in absence of strong anharmonic couplings, reasonable vibrational wavenumbers of stretching motions: this can be the case for $\mathrm{HC}_{3} \mathrm{~N}$. Also the comparison between VPT2 and DFT/MD values appears quite reasonable to describe the five highest modes (valence modes $\mathrm{v}_{1}$ to $\mathrm{v}_{4}$ and the bending mode $\mathrm{v}_{5}$ ) reported in Table 2, against the experimental data [23-25]. At the VPT2 level, the CCSD(T) wavenumbers are much closer to the experimental values than the DFT results. The mean average deviation varies from 11 to $48 \mathrm{~cm}^{-1}$. Molecular dynamic simulations show a relative weak influence of temperature with an average deviation ranging from $26 \mathrm{~cm}^{-1}(300 \mathrm{~K})$ to $19 \mathrm{~cm}^{-1}$ (600K). As a general rule, the CCSD(T)/VPT2 and B3LYP/MD frequencies lead to convergent results in fair agreement with the experimental data with a more favorable mean average deviation for the CCSD(T)/VPT2 static determination $\left(11 \mathrm{~cm}^{-1}\right)$ than for the B3LYP/MD dynamic one $\left(19 \mathrm{~cm}^{-1}\right)$.

\section{3-1 Propynenitrile in interaction with water.}

The study of 1:1 complexes of propynenitrile with water is undertaken to simulate as a first approximation the study of hydration and explain the expected vibration frequency shifts on the IR spectra. These calculated displacements will be compared to the experimental values from FT-IR matrix isolation spectroscopy reported by Borget et al [25]. For these 1:1 complexes, two structures are possible (fig 2). The most stable is the formation of a hydrogen bond between the oxygen in the water and the hydrogen of $\mathrm{HC}_{3} \mathrm{~N}(\mathrm{OH}$ structure), the other about $2 \mathrm{kcal} \mathrm{mol}^{-1}$ less stable combines the hydrogen in the water with the nitrogen in $\mathrm{HC}_{3} \mathrm{~N}(\mathrm{NH}$ structure). From a Boltzmann population point of view at $300 \mathrm{~K}$ the former represents $96 \%$. To best modelize the hydrogen bonds, calculations are also performed using the B3LYP-D/6-31G+(d,p) method. From a static point of view, the quartic force field is calculated on all complexes modes (molecule + water) but not by coupling for $\mathrm{HC}_{3} \mathrm{~N}$ the two lowest deformation modes $\left(\mathrm{v}_{6}\right.$ and $\left.\mathrm{v}_{7}\right)$ as for the study of the isolated molecule. From a dynamic 
point of view, DFT or DFT-D/MD calculations are performed with energies corresponding to temperatures around $300 \mathrm{~K}$ and $450 \mathrm{~K}$ for the $\mathrm{OH}$ structure where the largest band shifts relative to the isolated molecule are expected and around $300 \mathrm{~K}$ for the NH structure. All theoretical results are reported in Tables 3 and 4 for each of the complexes and compared to the experimental data reported by Borget et al [25].

When examining Tables 3 and 4, we can note a slight improvement brought by the consideration of dispersion effects (DFT-D better than DFT) as well as the relatively small influence of temperature in dynamic simulations with the exception of the $\mathrm{v}_{1}$ mode for the $\mathrm{OH}$ form largely affected by the hydrogen bond. The results provided by DFT-D/VPT2 and DFT-D/MD approaches are both qualitatively reliable, but globally, the mean average deviation (MAD) between theory and experiment is better for molecular dynamics (respectively 19 and $13 \mathrm{~cm}^{-1}$ for $\mathrm{OH}$ and $\mathrm{NH}$ forms) than for the static DFT/VPT2 approach (42 and $52 \mathrm{~cm}^{-1}$ respectively). However, we can emphasize that this difference is mainly due to the incorrect description of the $v_{2}$ mode in the DFT or DFTD/VPT2 computations. When we look at the displacements of the IR bands of the $\mathrm{OH}$ and NH forms relatively to the isolated molecule, we can see that the largest shifts concern the $\mathrm{OH}$ form. Indeed for this form the wavenumber of the $\mathrm{CH}$ valence mode $\left(\mathrm{v}_{1}\right)$ is downshifted by about hundred $\mathrm{cm}^{-1}\left(-98\right.$ to $-115 \mathrm{~cm}^{-1}$ according to our calculations) while the frequency of the $\mathrm{CCH}$ bending mode $\mathrm{v}_{5}$ is upshifted from 95 to $100 \mathrm{~cm}^{-1}$. These results are in excellent agreement with the experimental values reported by Borget et al $\left(-96\right.$ and $+93 \mathrm{~cm}^{-1}$ respectively). The large decrease observed for the $\mathrm{CH}$ stretching mode is likely due to the presence of hydrogen bond which increase the $\mathrm{CH}$ bond with respect to the isolated molecule, whereas the upshift of the bending mode is essentially due to the coupling between the bending mode $\mathrm{v}_{5}$ and the CC valence mode $\mathrm{v}_{4}$. The frequencies of the other modes are little modified by the presence of the hydrogen bond except for $\mathrm{v}_{3}$ upshifted of about ten $\mathrm{cm}^{-}$

${ }^{1}\left(13 \mathrm{~cm}^{-1}\right.$ in our calculations versus $10 \mathrm{~cm}^{-1}$ experimentally). For the $\mathrm{NH}$ structure, the shifts brought by solvation are lower and never exceed about ten $\mathrm{cm}^{-1}$ as confirmed by our calculations.

\section{Conclusion}

We have investigated the vibrational infra-red spectra of propynenitrile and its 1:1 complexes with water in the framework of quantum mechanics. Building on a B3LYP/6-31G+(d,p) level of theory, two different approaches have been tested to account for vibrational anharmonicity: a static calculation with second order vibrational perturbation theory (DFT/VPT2) and a dynamic through quantum molecular dynamics (DFT/MD). Comparing the results of these anharmonic quantum-mechanical approaches with experimental data reveals that if the 
perturbative treatment at the $\operatorname{CCSD}(\mathrm{T})$ /VPT2 level of theory offers a more reliable description of both stretching modes for the isolated molecule, the DFT/MD approach (extremely time consuming however) is better than the DFT/VPT2 (fastest when the quartic force constants are obtained) one, by using the same basis set, to describe the IR spectra of propynenitrile and its two 1:1 complexes with water. 


\section{References}

[1] E.B. Wilson, J.C. Decius, P.C. Cross, Molecular Vibrations Dover, New York, 1955

[2] P. Pulay, Modern Electronic Structure Theory, Part II, D. R. Yarkony, Ed., World Scientific, Singapore 1995 p. 1191, and references therein.

[3] A.P. Scott, L. Radom, Harmonic Vibrational Frequencies: An evaluation of Hartree-Fock, Moller-Plesset, Quadratic Configuration Interaction, Density Functional Theory and Semi-Empirical Scale Factors. J. Phys. Chem. 100 (1996) 16502-16513

[4] K.M.Dunn, J.E. Boggs, P. Pulay, Vibrational energy levels of hydrogen cyanide. J. Chem. Phys.85 (1986) 5838

[5] S.Carter, J.M. Bowman, In Rovibrational Bound States in Polyatomic Molecules M.M Law, I.A. Atkinson,

J.M. Hutson Eds , CCp6 Daresbury.1999

[6] P.Cassam-Chenaï, J. Lievin, The VMFCI method: A flexible tool for solving the molecular vibration problem. J. Comput.Chem. 27 (2006) 627-640

[7] F.Ribeiro, C. Iung, C. Leforestier, A Jacobi-Wilson description coupled to a block-Davidson algorithm: An efficient scheme to calculate highly excited vibrational levels. J. Chem. Phys. 123 (2005) 054106.

[8] D.Begue, N. Gohaud, C. Pouchan, P. Cassam Chenaï , J. Lievin, A comparison of two methods for selecting vibrational interaction spaces on a heptatomic system: Ethylene oxyde. J. Chem. Phys. 127 (2007) 164115. and references there in.

[9] S.L. Mielke, A. Chakraborty, D.G. Truhlar, Vibrational Configuration Interaction Using a Tiried Multimode Scheme and Tests of Approximate Treatments of Vibrational Angular Momentum Coupling : A case Study for Methane. J. Phys. Chem. A 117 (2013) 7327-7343

[10] C. Pouchan, K. Zaki, Ab Initio Configuration Interaction determination of the overtone vibrations of methyleneimine in the region 2800-3200 $\mathrm{cm}^{-1}$ J. Chem. Phys. 107 (1997) 342-345

[11] P. Carbonniere, A. Dargelos,C. Pouchan, The VCI-P code: an iterative variation scheme for efficient computations of anharmonic vibrational levels and IR intensities of polyatomic molecules. Theor. Chem. Accounts 125 (2010) 543-554

[12] G. Rauhut, Configuration selection as aroute towards efficient vibrational configuration interaction calculations. J. Chem. Phys. 127 (2007) 184109

[13] I.H. Godtliebsen, B. Thomsen, O. Christiansen, Tensor Decomposition and Vibrational Coupled Cluster Theory. J. Phys. Chem. A 117 (2013) 7267-7279

[14] N.K. Madsen, I.H. Godtliebsen, O. Christiansen, Efficient algorithms for solving the non-linear vibrational coupled-cluster equations using full and decomposed tensors. J. Chem. Phys.146 (2017) 134110. 
[15] J. Bloino, M. Biczysko, V. Barone, General Perturbative Approach for Spectroscopy, Thermodynamics, and Kinetics: Methodological Background and Benchmarks Studies. J. Chem. Theory. Comput. 8 (2012) 10151036 and references therein.

[16] P.H.Berens, K.R. Wilson, Molecular dynamics and spectra. I. Diatomic rotation and vibration. J. Chem. Phys. 74 (1981) 4872

[17] R. Ramirez, T. Lopez-Ciudad, P. Kumar, D. Marx, Quantum corrections to classical time-correlation functions: Hydrogen bonding and anharmonic floppy modes. J. Chem. Phys. 121 (2004) 3973.

[18] A.D Becke, Density-functional thermochemistry. III. The role of exact exchange; J. Chem. Phys. 98 (1993) 5648.

[19] P. Carbonniere, T. Lucca, N. Rega, C. Pouchan, V. Barone, Vibrational computations beyond the harmonic approximation: Performances of the B3LYP density functional for semi rigid molecules. J. Comput. Chem. 26 (2005) 384-388.

[20] S. Uyemura, S. Degushi, Y. Nakada, T. Onaka, Infrared intensities of Bending Fundamentals in Gaseous HCCCN and DCCCN. Bull. Chem. Soc. Japan. 55 (1982) 384-388

[21] F. Khlifi, E. Raulin, E. Arié, G. Graner, Absolute Intensity of the IR Bands of Propynenitrile. J.Mol. Spectrosc. 143 (1990) 209-211

[22] F.Khlifi, E. Raulin, M. Dang-Nhu, Integrated band intensity versus temperature for $v_{1}, v_{2}, v_{5}$, and $v_{6}$ bands of cyanoacetylene. J.Mol. Spectrosc. 155 (1992) 77-83

[23] A.Jolly, Y. Benilan, A. Fayt, New infrared integrated bands intensities for $\mathrm{HC}_{3} \mathrm{~N}$ and extensive line list for the $v_{5}$ aand $v_{6}$ bending modes. J.Mol. Spectrosc. 242 (2007) 46-54

[24] L. Bizzochi, F. Tamassi, J. Laas, B.M.Guiliano, C. Degli Esposti, L. Dore, M. Melosso, E. Canè, A. Pietropolli Charmrt, S.P. Muller Holger, Holger Spahn, A. Belloche, P. Caselli, K.M. Menten, R.T. Garrod, Rotational and High-resolution Infrared Spectrum of $\mathrm{HC}_{3} \mathrm{~N}$ : Global Ro-vibrational Analysis and Improved Line Catalog for Astrophysical Observations. The Astrophysical Supplement Series 233 (2017) 11 (1-20)and references therein.

[25] F. Borget, T. Chiavassa, A. Allouche, F. Marinelli, J.P. Aycard, Cyanoacetylene Adsorption on Amorphous and Crystalline Water Ice Films: Investigation through Matrix Isolation and Quantum Study. J. Am. Chem. Soc. 123 (2001) 10668-10675

[26] M.J. Frisch, G.W. Trucks, H.B. Schlegel, G.E. Scuseria, M.A. Robb, J.R. Cheeseman, G. Scalmani, V.

Barone, B. Menucci, G.A. Petterson, et al., (2009) Gaussian 09, Revision D.1; Gaussian Inc: Wallingford CT.

[27] P. Carbonniere, A. Dargelos, I. Ciofini, C. Adamo, C.Pouchan, Vibrational analysis of glycine radical: a comparative ab initio static and dynamic study Phys. Chem.Chem. Phys 11 (2009) 4375-4384 
[28] P. Carbonniere, V. Barone, Performances of different density functionals in the computation of vibrational spectra beyond the harmonic approximation. Chem Phys Lett 399 (2004) 226-229

[29] P. Carbonniere, C. Pouchan, Vibrational spectra for $\mathrm{P}(4) \mathrm{O}(6)$ and $\mathrm{P}(4) \mathrm{O}(10)$ systems: Theoretical study from DFT quartic potential and mixed perturbation-variation method. Chem Phys Lett 462 (2008) 169-172 [30] V. Barone, P. Carbonniere, C. Pouchan, Accurate vibrational spectra and magnetic properties of organic

free radicals: The case of H2CN. J. Chem. Phys.122 (2005) 2443081

[31] K. Ragavarachri, G.W. Trucks, J.A. Pople, M. Head- Gordon, A fifth-order perturbation comparison of electron correlation theories. Chem. Phys. Lett. 157 (1989) 479-483

[32] R.A.Kendall, T.H. Dunning, R.J. Harrison, Electron affinities of the first- row atoms revisited. Systematic basis sets and wave functions. J. Chem. Phys. 96 (1992) 6796.

[33] P. Carbonniere, D. Begue, A. Dargelos, C. Pouchan, Construction of an accurate quartic force field by using generalized least-square fitting and experimental design. Chem. Phys. 300 (2004) 41-51

[34] W.H. Miller, R. Hernandez, N.C. Handy, D. Jayatilaka, A. Willets, Ab initio calculation of anharmonic constants for a transition state, with application to semi classical transition state tunneling probabilities. Chem. Phys. Lett. 172 (1990) 62-68

[35] V. Barone, Anharmonic vibrational properties by a fully automated second-order perturbative approach.

J. Chem. Phys. 122 (2005) 014108.

[36] H.B. Schlegel, J.M. Millam, S.S. Iyengar, G.A. Voth, A.D. Daniels, G.E. Scuseria, M.J. Frisch, Ab initio molecular dynamics: Propagating the density matrix with Gaussian orbitals. J. Chem. Phys. 114 (2001) 9758.

[37] S.S. Iyengar, H.B. Schlegel, J.M. Millam, G.A. Voth, G.E. Scuseria, M.J. Frisch, Ab initio molecular dynamics: Propagating the density matrix with Gaussian orbitals. II. Generalizations based on mass-weighting, idempotency, energy conservation and choice of initial conditions. J. Chem. Phys. 115 (2001) 10291.

[38] H.B. Schlegel, S.S Iyengar, X. Li, J.M. Millam, G.A. Voth, G.E. Scuseria, M.J. Frisch, Ab initio molecular dynamics: Propagating the density matrix with Gaussian orbitals. III. Comparison with Born-Oppenheimer dynamics. J. Chem. Phys. 117 (2002) 8694.

[39] M. Pavone, V. Barone, I. Ciofini, C. Adamo, First-principle molecular dynamics of the Berry pseudorotation: Insights on 19F NMR in SF4. J. Chem. Phys. 120 (2004) 9167.

[40] L. Joubert, C. Adamo, Static and dynamic descriptions of bond breaking/formation: A complementary view? J. Chem. Phys. 123 (2005) 211103.

[41] I. Ciofini, C. Adamo, Static and Dynamic approaches for the calculation of NMR parameters:

Permanganate ion as a case study. J. Mol. Struct. (THEOCHEM) 762 (2006) 133-137

[42] M. Martinez, M.P. Gaigeot, D. Borgis, R. Vuilleumier Extracting effective normal modes from equilibrium dynamics at finite temperature. J. Chem. Phys. 125 (2006) 144106. 
[43] M. Schmitz, P. Tavan, Vibrational spectra from atomic fluctuations in dynamics simulations: I. Theory, limitations, and a sample application. J. Chem. Phys. 121 (2004) 12233-12246.

[44] M. Schmitz, P. Tavan, Vibrational spectra from atomic fluctuations in dynamics simulations. II. Solventinduced frequency fluctuations at femtosecond time resolution. J. Chem. Phys. 121 (2004) 12247-12258

[45] J.A. Boatz, M.S. Gordon, Decomposition of normal-coordinate vibrational frequencies.

J. Phys. Chem. 93 (1989) 1819-1826.

[46] P. Carbonniere, S. Thicoipe, T. Very, X. Assfeld, Vibrational analysis beyond the harmonicity from Ab initio molecular dynamics: Case of cytosine in its anhydrous and aqueous forms. Int. J. Quantum. Chem 112 (2012) 2221

[47] S. Thicoipe, P. Carbonniere, C. Pouchan, Structural investigation of microhydrated thymine clusters and vibrational study of isolated and aqueous forms of thymine using DFT level of theory. Phys. Chem. Chem. Phys. 15 (2013) 11646-11652

[48] S. Thicoipe, P. Carbonniere, C. Pouchan, Comparison of static and dynamic methods of treatment of anharmonicity for the vibrational study of isolated and aqueous forms of guanine. Chem. Phys. Letters 591 (2014) 243-247

[49] S. Thicoipe, P. Carbonniere, C. Pouchan, DFT modelling of the infrared spectra for isolated and aqueous forms of adenine. Theoretical Chemistry Accounts 136 (2018) 44

[50] C. Pouchan, S. Thicoipe, M. De La Pierre, DFT modelling of the infrared spectra for the isolated and the

micro-hydrated forms of uracil. Theoretical Chemistry Accounts 138 (2019) 36 DOI10.1007/s00214-019-

24314.

[51] R. Burel, S. Carter, N.C. Handy, On the representation of potential energy surfaces of polyatomic molecules in normal coordinates. II Parametrisation of the force field. Chem. Phys. Letters 373 (2003) 357

TABLE 1: Computed harmonic wavenumbers (in cm-1) for propynenitrile in vacuo at different levels of theory. Comparison with experimental values

\begin{tabular}{|c|c|c|c|c|l|}
\hline Mode & $\begin{array}{c}\text { Main } \\
\text { Assignment }\end{array}$ & $\begin{array}{c}\text { CCSD(T)/ } \\
\text { aug-cc- } \\
\text { pVTZ }\end{array}$ & $\begin{array}{c}\text { CCSD / } \\
\text { Aug-cc- } \\
\text { pVTZ }\end{array}$ & $\begin{array}{c}\text { B3LYP /6- } \\
31+G(d, p)\end{array}$ & Experimental \\
\hline & & $\omega$ & $\omega$ & $\omega$ & $v$ \\
\hline$v_{7}$ & $\delta(\mathrm{C}-\mathrm{CC}-\mathrm{N})^{\mathrm{a}}$ & 218 & 226 & 244 & $222^{\mathrm{c}}$ \\
\hline$v_{6}$ & $\delta(\mathrm{CCN})^{\mathrm{a}}$ & 487 & 509 & 563 & $\begin{array}{l}504^{\mathrm{d}} ; 499^{\mathrm{c}} ; \\
500^{\mathrm{b}}\end{array}$ \\
\hline$v_{5}$ & $\delta(\mathrm{CCH})^{\mathrm{a}}$ & 664 & 702 & 706 & $667^{\mathrm{d}} ; 663^{\mathrm{c}} ; 664^{\mathrm{b}}$ \\
\hline$v_{4}$ & $v(\mathrm{C}-\mathrm{C})$ & 875 & 883 & 907 & $862^{\mathrm{c}} ; 861^{\mathrm{b}}$ \\
\hline$v_{3}$ & $v(\mathrm{C} \equiv \mathrm{C})$ & 2100 & 2164 & 2174 & $2077^{\mathrm{d}} ; 2079^{\mathrm{c}}$ \\
\hline$v_{2}$ & $v(\mathrm{CN})$ & 2306 & 2393 & 2374 & $2269^{\mathrm{d}} ; 2274^{\mathrm{c}}$ \\
\hline$v_{1}$ & $v(\mathrm{CH})$ & 3445 & 3472 & 3474 & $3316^{\mathrm{d}} ; 3327^{\mathrm{c}}$ \\
\hline
\end{tabular}

${ }^{a}$ Double degenerated modes 
${ }^{b} \operatorname{ref}[24] ;{ }^{c} \operatorname{ref}[23] ;{ }^{\mathrm{d}} \operatorname{ref}[25]$

TABLE 2: Wavenumbers (in cm-1) calculated for prpynenitrile in vacuo following VPT2 and DFT/MD approaches. Comparison with experimental values

\begin{tabular}{|c|c|c|c|c|c|c|}
\hline Mode & $\begin{array}{c}\text { VPT2 }^{\mathrm{a}}: \text { CCSD(T)/ } \\
\text { aug-cc-pVTZ }\end{array}$ & $\begin{array}{c}\text { VPT2 : } \\
\text { B3LYP /6- } \\
31+\mathrm{G}(\mathrm{d}, \mathrm{p})\end{array}$ & \multicolumn{3}{|c|}{ DFT/MD } & Experiment \\
\hline & $\boldsymbol{V}$ & $\boldsymbol{v}$ & $\mathrm{T}=300 \mathrm{~K}$ & $\mathrm{~T}=450 \mathrm{~K}$ & $\mathrm{~T}=600 \mathrm{~K}$ & $v$ \\
\hline$v_{7}$ & $218^{\mathrm{a}}$ & $244^{\mathrm{a}}$ & 252 & 251 & 248 & $222^{\mathrm{c}}$ \\
\hline$v_{6}$ & $487^{\mathrm{a}}$ & $563^{\mathrm{a}}$ & 567 & 559 & 563 & $\begin{array}{c}504^{\mathrm{d}} ; 499^{\mathrm{c}} ; \\
500^{\mathrm{b}}\end{array}$ \\
\hline$v_{5}$ & 657 & 698 & 652 & 652 & 657 & $\begin{array}{c}667^{\mathrm{d}} ; 663^{\mathrm{c}} ; \\
664^{\mathrm{b}}\end{array}$ \\
\hline$v_{4}$ & 867 & 897 & 903 & 900 & 903 & $862^{\mathrm{c}} ; 861^{\mathrm{b}}$ \\
\hline$v_{3}$ & 2078 & 2145 & 2093 & 2088 & 2083 & $2077^{\mathrm{d}} ; 2079^{\mathrm{c}}$ \\
\hline$v_{2}$ & 2279 & 2344 & 2252 & 2247 & 2242 & $2269^{\mathrm{d}} ; 2274^{\mathrm{c}}$ \\
\hline$v_{1}$ & 3337 & 3365 & 3363 & 3354 & 3346 & $3316^{\mathrm{d}} ; 3327^{\mathrm{c}}$ \\
\hline
\end{tabular}

${ }^{\text {a }}$ Order 2 : CCSD(T)/aug-cc-pVTZ - Orders 3 and 4 : B3LYP/6-31+G(d,p)

Modes $v_{7}$ and $v_{6}$ are totally decoupled from others: their harmonic values are reported in the table 2

${ }^{\mathrm{b}} \operatorname{Ref}$ [24]; ${ }^{\mathrm{c}} \operatorname{Ref}$ [23]; ${ }^{\mathrm{d}} \operatorname{Ref}[25]$

TABLE 3: Wavenumbers (in cm-1) calculated following VPT2 and DFT/MD approaches for HC3N:H20 (OH form). Comparison with experimental values

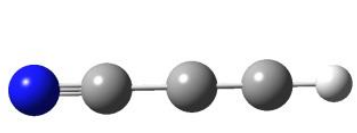

\begin{tabular}{|c|c|c|c|c|c|c|c|c|}
\hline \multirow{2}{*}{ Mode } & \multicolumn{4}{|c|}{ VPT2/B3LYP } & \multicolumn{3}{c|}{ DFT or DFT-D/MD } & Experiment $^{\mathrm{a}}$ \\
\cline { 2 - 9 } & $\omega_{\text {DFT }}^{\mathrm{b}}$ & $v_{\text {DFT }}$ & $\begin{array}{c}\omega_{\text {DFT- }} \\
\mathrm{c}^{\mathrm{n}}\end{array}$ & $v_{\text {DFT-D }}$ & $\begin{array}{c}\text { DFT } \\
450 \mathrm{~K}\end{array}$ & $\begin{array}{c}\text { DFT- } \\
\text { D } \\
450 \mathrm{~K}\end{array}$ & $\begin{array}{c}\text { DFT-D } \\
300 \mathrm{~K}\end{array}$ & \\
\hline$v_{7}$ & 307 & - & 306 & & 260 & 260 & 282 & \\
\hline$v_{6}$ & 646 & - & 645 & & 538 & 540 & 555 & 506 \\
\hline$v_{5}{ }^{\mathrm{e}}$ & 874 & - & 879 & - & 738 & 742 & 752 & 760 \\
\hline$v_{4}$ & 910 & 901 & 906 & 894 & 899 & 895 & 900 & \\
\hline$v_{3}$ & 2161 & 2132 & 2157 & 2116 & 2090 & 2088 & 2085 & 2067 \\
\hline
\end{tabular}




\begin{tabular}{|c|c|c|c|c|c|c|c|c|}
\hline$v_{2}$ & 2367 & 2330 & 2364 & 2324 & 2251 & 2252 & 2253 & 2263 \\
\hline$v_{1}$ & 3354 & 3220 & 3339 & 3201 & 3294 & 3294 & 3248 & 3219 \\
\hline MAD. $^{d}$ & & 44 & & 42 & 37 & 36 & 19 & \\
\hline
\end{tabular}

${ }^{a}$ Ref [25]

${ }^{\mathrm{b}}$ B3LYP/6-31+G(d,p) calculations

${ }^{\mathrm{c}}$ B3LYP-D/6-31+G(d,p) calculations

${ }^{\mathrm{d}}$ MAD. for Mean Average Deviation (in $\mathrm{cm}^{-1}$ ) for the valence modes only

${ }^{\mathrm{e}}$ Not converged values

TABLE 4: Wavenumbers (in cm-1) calculated following VPT2 and DFT-D/MD approaches for HC3N:H20 (NH form). Comparison with experimental values
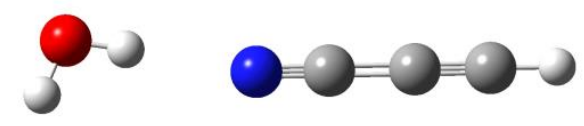

\begin{tabular}{|c|c|c|c|c|}
\hline \multirow{2}{*}{$\begin{array}{c}\text { Mode } \\
\text { Assignment }\end{array}$} & \multicolumn{2}{|c|}{ VPT2 } & Molecular Dynamics & Experiment $^{\text {a }}$ \\
\cline { 2 - 5 } & $\begin{array}{c}\omega_{\text {DFT- }} \\
\text { D }\end{array}$ & $\begin{array}{c}v_{\text {DFT- }} \\
\text { D }\end{array}$ & DFT-D 300K & \\
\hline$v_{7}$ & 232 & - & 221 & \\
\hline$v_{6}$ & 568 & - & 572 & 670 \\
\hline$v_{5}$ & 719 & 706 & 680 & \\
\hline$v_{4}$ & 910 & 901 & 903 & 2273 \\
\hline$v_{3}$ & 2176 & 2146 & 2097 & 3312 \\
\hline$v_{2}$ & 2380 & 2340 & 2258 & \\
\hline$v_{1}$ & 3463 & 3342 & 3367 & \\
\hline MAD. & & 52 & 13 & \\
\hline
\end{tabular}

${ }^{a} \operatorname{Ref}[25]$

${ }^{\mathrm{b}}$ B3LYP-D/6-31+G(d,p) calculations

${ }^{\mathrm{c}}$ MAD. for Mean Average Deviation (in $\mathrm{cm}^{-1}$ )

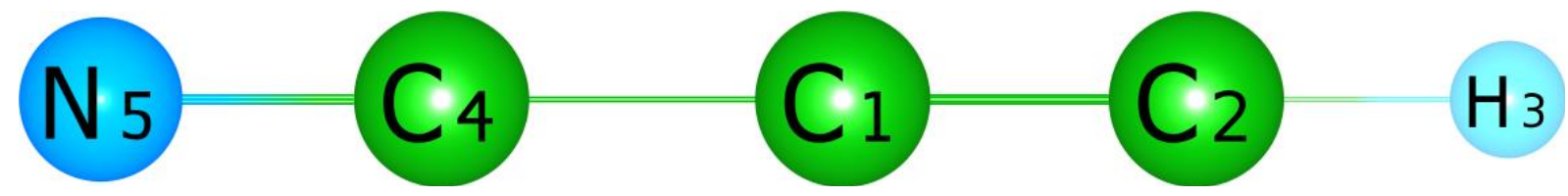

Fig 1 : Propynenitrile atomic numbering 

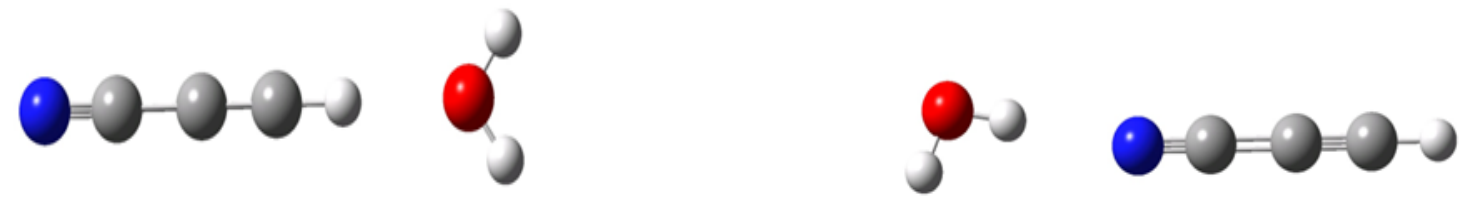

Fig 2: $\mathrm{OH}$ and $\mathrm{NH}$ forms of the $1: 1$ complex between $\mathrm{HC}_{3} \mathrm{~N}$ and $\mathrm{H}_{2} \mathrm{O}$ 
Caption for Tables:

TABLE 1: Computed harmonic wavenumbers (in cm-1) for propynenitrile in vacuo at different levels of theory. Comparison with experimental values

TABLE 2: Wavenumbers (in cm-1) calculated for propynenitrile in vacuo following VPT2 and DFT/MD approaches. Comparison with experimental values

TABLE 3: Wavenumbers (in cm-1) calculated following VPT2 and DFT/MD approaches for

$\mathrm{HC}_{3} \mathrm{~N}: \mathrm{H}_{2} \mathrm{O}$ (OH form). Comparison with experimental values

TABLE 4: Wavenumbers (in cm-1) calculated following VPT2 and DFT-D/MD approaches for HC N:H O (NH form). Comparison with experimental values 32

Caption for Figures:

Fig 1 : Propynenitrile atomic numbering

Fig 2: $\mathrm{OH}$ and $\mathrm{NH}$ forms of the $1: 1$ complex between $\mathrm{HC}_{3} \mathrm{~N}$ and $\mathrm{H}_{2} \mathrm{O}$ 
Graphical Abstract:

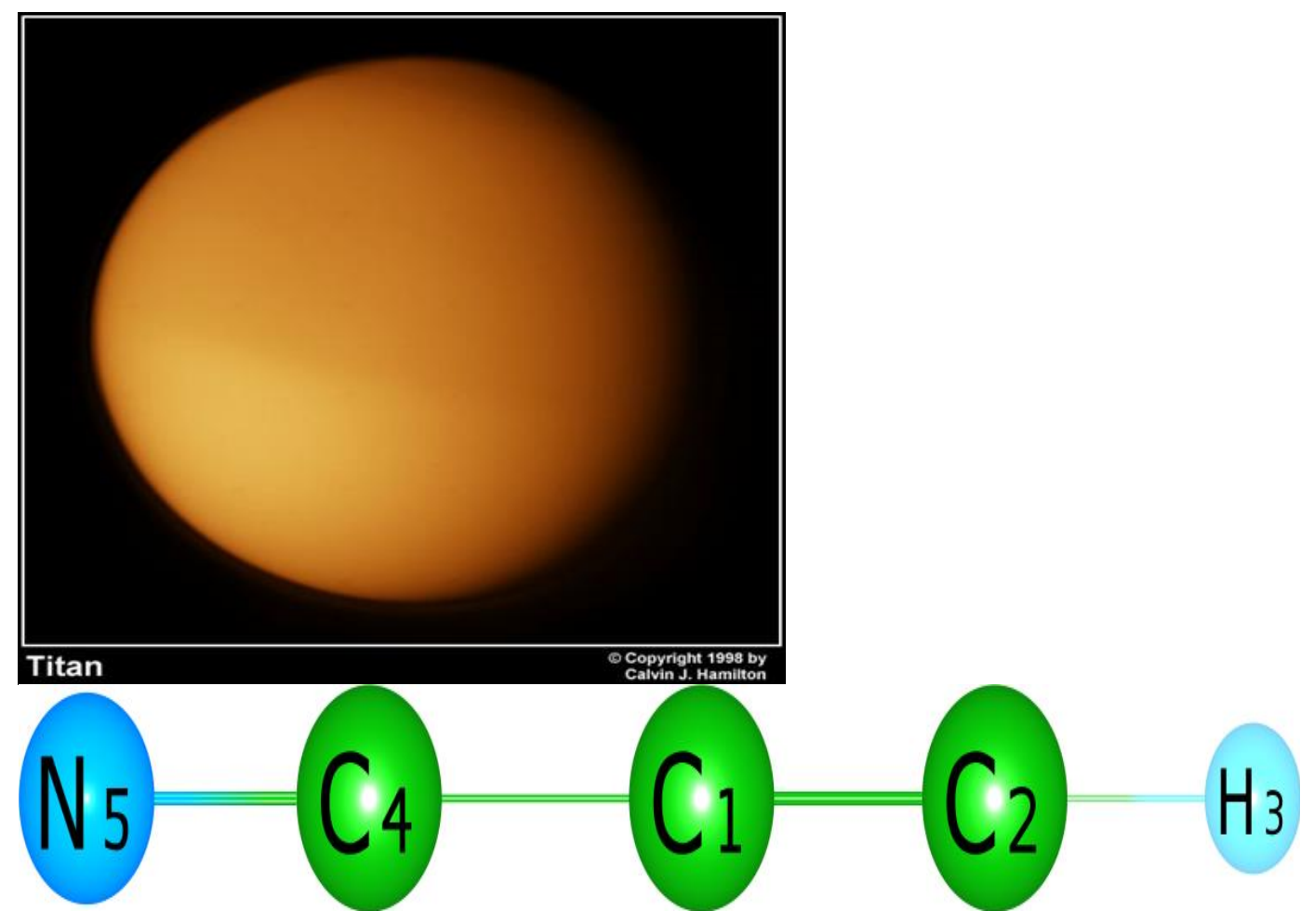

\section{Highligths}

- Propynenitrile $\mathrm{HC}_{3} \mathrm{~N}$ is a molecule of astronomical importance detected in interstellar clouds

- We compare static (VPT2) and dynamic (DFT/MD) approaches to modelize the IR spectrum in vacuo.

- IR spectra of 1:1 complexes with water are studied by DFT/MD to analyze the shifts expected by the environment 